\title{
Effect of checklist based box system interventions on improving institutional delivery among reproductive age women in Northwest Ethiopia: generalized structural equation modeling
}

\author{
Netsanet Belete Andargie ${ }^{1,2^{*}}$ (D) and Gurmesa Tura Debelew ${ }^{2}$
}

\begin{abstract}
Background: Previous studies have shown that there is low utilization of institutional delivery in Ethiopia, as well as various factors contributing to this low utilization. Notably, there is paucity around interventions to improve institutional delivery. Hence, this study examines the effectiveness of checklist-based box system intervention on improving institutional delivery and to investigate the association through which the intervention is linked to institutional delivery.

Method: The study used data from a larger trial, on the effectiveness of checklist-based box system intervention on improving maternal health service utilization. In the intervention arm, mothers received regular community-level pregnancy screening and referral, service utilization monitoring boxes, drop-out tracing mechanisms, regular communication between health centers and health posts, and person-centered health education for mothers. This study used the existing government-led maternal health program as a control arm. A total of 1062 mothers who gave birth one-year before the survey were included in the final analysis. A difference-in-difference estimator was used to test the effectiveness of the intervention. Generalized structural equation modeling was used to examine the direct and/ indirect associations between the intervention and institutional delivery.

Result: Among participants, 403 (79.5\%) mothers from intervention and 323 (58.2\%) mothers from control clusters gave birth at health facilities. The result of the study revealed a 19\% increase in institutional delivery in the intervention arm (19,95\% Cl: 11.4-27.3\%). In this study the pathway from checklist-based box system intervention to institutional delivery was mainly direct - (AOR $=3.32,95 \% \mathrm{Cl}$ : 2.36-4.66), however, $33 \%$ of the effect was partially mediated by attendance of antenatal care four visits ( $A O R=1.39,95 \% \mathrm{Cl}$ : 1.02-1.92). The influence of significant others (AOR $=0.25,95 \% \mathrm{Cl}: 0.15-0.43)$ and age ( $\mathrm{AOR}=0.03,95 \% \mathrm{Cl}: 0.01-0.09)$ had an inverse relation with institutional delivery.
\end{abstract}

\footnotetext{
* Correspondence: netsanetb2009@gmail.com

${ }^{1}$ Ministry of Health, Addis Ababa Ethiopia and Department of Population and Family Health, Jimma University, Jimma, Ethiopia

${ }^{2}$ Department of Population and Family Health, Jimma University, Jimma, Ethiopia
}

(c) The Author(s). 2021 Open Access This article is licensed under a Creative Commons Attribution 4.0 International License, which permits use, sharing, adaptation, distribution and reproduction in any medium or format, as long as you give appropriate credit to the original author(s) and the source, provide a link to the Creative Commons licence, and indicate if changes were made. The images or other third party material in this article are included in the article's Creative Commons licence, unless indicated otherwise in a credit line to the material. If material is not included in the article's Creative Commons licence and your intended use is not permitted by statutory regulation or exceeds the permitted use, you will need to obtain permission directly from the copyright holder. To view a copy of this licence, visit http://creativecommons.org/licenses/by/4.0/. The Creative Commons Public Domain Dedication waiver (http://creativecommons.org/publicdomain/zero/1.0/) applies to the data made available in this article, unless otherwise stated in a credit line to the data. 
Conclusion: The implementation of a checklist-based box system significantly increased institutional delivery utilization, both directly and indirectly by improving antenatal care four attendance. A larger-scale implementation of the intervention was recommended, taking the continuum of care approach into account.

Trial registration: ClinicalTrials.gov, NCT03891030, Retrospectively registered on 26 March, 2019.

Keywords: Box system, Institutional delivery, Generalized structural equation modeling, Northwest Ethiopia

\section{Background}

The World Health Organization (WHO) recommends that all pregnant women receive quality care throughout their pregnancy, childbirth, and postnatal period. WHO prioritized emergency obstetric care in its safe motherhood initiative, with the goal of ensuring safe birth and reducing actual and potential complications for the mother and newborn [1-4]. Studies showed that institutional delivery could reduce the occurrence of maternal death by $16-33 \%[5,6]$. However, in developing countries most deliveries occur at home without skilled care assistance [7, 8]. Evidence from the 2019 Ethiopian Health and Demographic Survey (EDHS) showed that only $48 \%$ of women went to health facilities for childbirth services [9].

Institutional delivery was influenced by previous maternal health care visits that a mother had [10]. Studies also recommend that, unless antenatal care (ANC) becomes a bridge for having institutional deliveries, it may not fully achieve its objective as major life-threatening obstetric complications commonly occur during and after delivery [11, 12]. Previous studies in Ethiopia investigate the magnitude of institutional delivery, and most reported it as 'low' [1316], compared to the country level goal for 2020, which was 90\% [17]. Works of literatures also identified several modifiable factors associated with institutional delivery such as attitude towards institutional delivery, maternal age, frequency of antenatal care visits, antenatal depressive symptoms, attending pregnant mother conferences etc. However, in Ethiopia, there are limited interventional studies conducted to improve utilization of institutional delivery. A pragmatic study on the effectiveness of Maternity waiting homes (MWHs), which are home-like environments located near health centers for near-term pregnant mothers, combined with community mobilization by trained local leaders in improving institutional births, revealed a small but non-significant improvement in institutional delivery [18]. Another intervention on the effect of $m$-health intervention on institutional delivery and postnatal care utilization involves, reminders of subsequent visits of ANC, delivery and PNC as well as educational messages on dangers signs during pregnancy and common complaints during pregnancy. This message in the pregnancy period was discovered to improve utilization of institutional delivery and postnatal care [19]. Another community trial to improve institutional delivery is the deployment of trained community-based nurses to rural communities in Ethiopia to assess SBA uptake levels. This study found that sending trained reproductive health nurses to rural Ethiopian communities increased SBA service utilization significantly [20].

Hence, checklist based box system intervention (CBBSI) was designed with the aim of contributing to improved maternal health care utilization, including institutional delivery. The intervention focused on the continuum of maternal health care and assessed the impact of previous maternal health visits (attendance of antenatal care four visit) on institutional delivery. This can also be implemented in both rural and urban settings, regardless of mobile phone ownership. The intervention was implemented with a twopronged approach of demand creation and service utilization monitoring through drop-out tracing mechanisms. The intervention provided community-level pregnancy screening to identify suspected pregnant women and link them to a nearby health center. After the pregnancy was confirmed at the health center, the mother received her first ANC and became a part of the "service utilization monitoring box," which was designed to track service utilization from the first antenatal care visit to the third postnatal care visit. This service utilization monitoring box assists midwives in identifying mothers who fail to attend recommended maternal health care visits. Through health education, health extension workers, in which the dropping mother belong, were able to sway her to return to service. The "health education scheduling box" aided health extension workers in prioritizing health education topics during this process [21].

In this study, the effectiveness of checklist based box system interventions on improving utilization of institutional delivery was assessed. In addition, the contribution of previous maternal health care visits including frequency of antenatal care visit to institutional delivery have been reported by many studies [14, 15, 22-24]. Therefore, this study also assessed the indirect paths through which the intervention is linked to institutional delivery. 


\section{Methods}

This trial was retrospectively registered on ClinicalTrials.gov with trial identifier NCT03891030 on March 26, 2019, and the trial protocol was published [21].

\section{Study design and setting}

A double-blind, parallel-group, two-arm cluster randomized controlled trial was conducted to assess the effectiveness of checklist-based box system interventions on improving utilization of institutional delivery. The study was conducted in three districts of East Gojjam Zone: Debremarkos, Gozamin, and Machakel. East Gojjam zone is one of the administrative Zones of the Amhara region located in Northwest Ethiopia. According to the 2007 census, in the Amhara region, the total population was 2,153,937 and of these 1,087,221 were female [25]. In the region, $82.6,55.7,39.8 \%$ of mothers received at least one antenatal care, institutional delivery, and postnatal care, respectively [9]. Currently in Ethiopia, focused antenatal care (four visit model) is being implemented at the national level. This study was conducted from January 2019-September 2020.

\section{Study population and inclusion criteria}

The study considers mothers of reproductive age group (15-49 years) as a source population. Similarly, mothers who gave birth last 1 year preceding the survey and part of checklist-based box system intervention trial were included.

\section{Sample size determination and sampling procedure}

To get a more stable parameter estimates, the sample size for this study was calculated based on the estimable parameter to respondent ratio of 1:30 [26]. Accordingly, the number of estimable parameters were 26, which made the total sample size required to be 780 . However, as this study is part of a larger trial, data from 1062 participants were included in the final analysis.

Regarding the sampling procedure, of the 16 districts available in East Gojjam Zone: Debre-Markos, Gozamin, and Machakel were selected on the basis of confirming that health posts/health centers within these districts didn't receive an intervention/project aiming to improve utilization of maternal health services. Then a random sampling method was used to select health posts that were included in the study. The list of mothers who gave birth within 1 year preceding the survey was accessed from the health post's family folder, where the profile of the residents in the catchment area of the health post was registered in. Then this list was entered into SPSS and taken as a sampling frames for the study. Then the required numbers of mothers were selected from the list using SPSS random selection command.

\section{Intervention}

Checklist-based box system intervention is a service utilization improvement project aiming to improve the utilization of maternal health services: antenatal care, institutional delivery, and postnatal care. The intervention consisted of both demand creation and service utilization monitoring components. Mothers below 16 weeks of gestation were enrolled in the study and followed until they reached their postnatal care three visits. In this process, mothers who belong to the intervention group received: community-level pregnancy screening, then suspected pregnant mothers were linked with the nearby health center for laboratory confirmation of the pregnancy. Mothers with confirmed pregnancy received the first ANC on the same day, then service utilizations were monitored for each and every mother using a service utilization monitoring box placed at health centers. Mothers who drop the service before getting the third postnatal care had a reminder visit by health extension workers to resume their visits. The second box was placed at health posts where demand creation activities were scheduled. Health education topics were determined based on the gap that health extension workers identified during their community survey. The detailed procedure of the intervention process can be accessed from the published study protocol [21].

\section{Variables and measurement}

- Institutional Delivery - Participants were asked 'where their last childbirth took place?' Response were ' 0 for Home' or ' 1 for Health Facility'.

- Month of ANC initiation - participants were asked about the time they initiated their ANC visits/ receive the first antenatal care, and responses were less than or equal to 16 weeks and greater than 16 weeks of gestation.

- Antenatal care four visits- Participants were asked 'How many antenatal care visits did they attend for their index pregnancy?' This was recoded as 'four or more visits' and 'less than four visits'. Institutional delivery, month of ANC initiation and antenatal care four visits were treated as endogenous observed variables in the generalized structural equation model.

- Checklist Based Box system Intervention - This was examined as whether the participant belongs to an intervention or a control wing.

- Social Support - Social support was measured using the following 14 item questions with 'yes' or 'no' response categories: 'gets visits from significant others', 'getting useful advises', 'discussion on problems', 'having care at the time of labor and delivery', 'feeling loved', 'others thankful on them', 
'getting help on household chores', 'help with money at emergency', 'help in transportation', 'help when sick', 'attending community level discussions', 'member of any religious cast', 'attending public meetings' and 'help in case of conflicts'.

- Influence - Participants were asked whether they had a negative influences from significant others (mother, grandmother, mother, neighbors, and husband) in the process of using maternal health services. The responses were 'yes' or 'no'. Checklist based box system intervention, social support and influence were treated as exogenous observed variables in the generalized structural equation model.

- Knowledge of danger signs of labor and delivery this was measured using an item consisting of seven questions with 'yes' or 'no' response categories: 'severe headache', 'vaginal bleeding', 'convulsion', 'high fever', 'loss of consciousness', 'retained placenta' and 'prolonged labor'.

- Birth preparedness and complication readiness - was examined as a latent construct consisting of five items with 'yes' or 'no' response category: 'arranged emergency transport', 'arranged emergency funds', 'personal saving and how to access them', 'choose place of delivery/skilled attendant' and 'knows who the blood donor is'. Birth preparedness and complication readiness and knowledge of danger signs of pregnancy and delivery were treated as endogenous latent variables.

- Family Support - was examined as a latent construct consisting of five item questions with 'yes' or 'no' response categories: 'Provided advice and support to deliver at health facilities', 'help to get emergency transport during labor', 'accompany during labor', 'help to care baby', and 'help to attend post-natal visits'. Family support was treated as exogenous latent variable.

\section{Hypothesized theoretical model}

The hypothesized relationship between observed and latent variables with the outcome were constructed based on the findings from the literature review, the practicability of relationships, and authors' experience. Then, potential pathways through which the intervention is linked to institutional delivery were created (Fig. 1).

Previous studies reported that mothers attending four ANC visits were high likely to deliver at health facilities $[14,15,22-24,27,28]$. In addition to the direct relationship between the intervention and institutional delivery, pathways were created through potential mediators to institutional delivery (Fig. 2). In addition, studies conducted in South West and South East Ethiopia [8, 29] identified knowledge on danger signs of labor and delivery as one of the determinant factors for institutional delivery, similarly, mothers having birth preparedness and complication readiness practice more likely went for facility delivery as compared to their counterparts [24].

\section{Data processing, model building and analysis}

Data was exported from Kobo Toolbox and imported to STATA Version 15 for analysis. Before the analysis, data were checked for missing values and outliers. Descriptive analysis was conducted to understand sample characteristics. Then, statically significant variables were identified by using bivariate analysis. The effectiveness of checklist-based box system intervention in improving institutional delivery was tested by using the difference in difference estimator.

Factor analysis was used to reduce the number of items per latent construct and to determine the contribution of each observed variable to the latent construct. Then, confirmatory factor analysis was used to see if the data fit the hypothesized measurement model and if there was a relationship between the observed variables and their underlying latent constructs. Accordingly, birth preparedness and complication readiness practice,

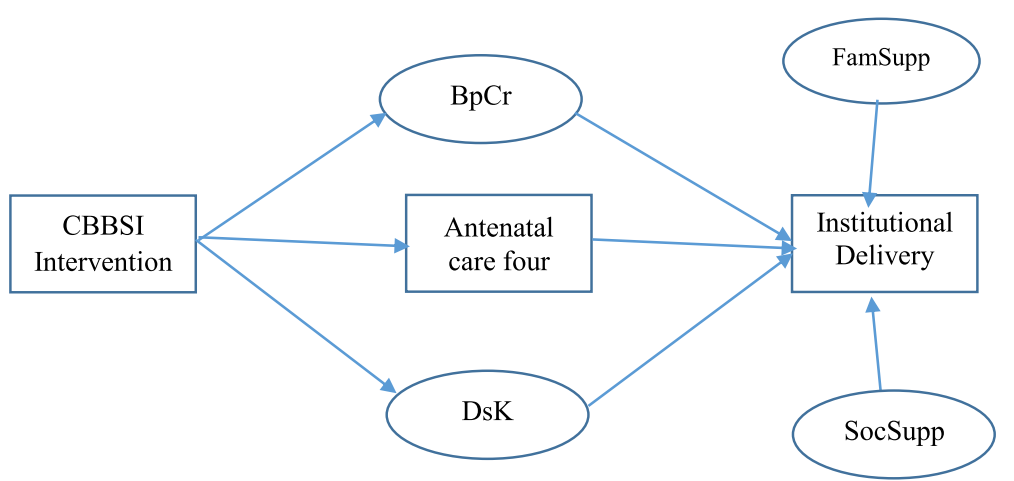

Fig. 1 Hypothesized model for factors associated with institutional delivery: \{Adjusted for age, month of initiation for the first ANC, influence\}, January 2019-September 2020, Northwest Ethiopia (CBBSI-Checklist based box system intervention, BpCr-Birth Preparedness and complication readiness, DsK-Danger Signs Knowledge, FamSupp-Family support) 

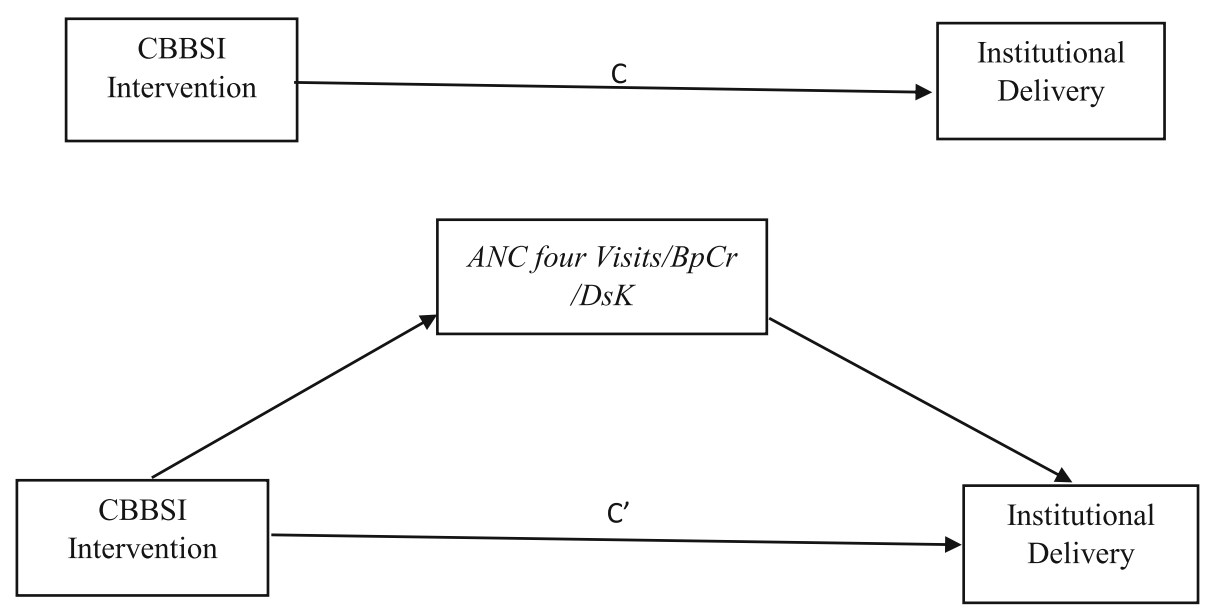

Fig. 2 The effect of CBBSI on institutional delivery and the mediation effect of Antenatal four visits, birth preparedness and complication readiness and danger signs of labor and delivery, January 2019-September 2020, Northwest Ethiopia. (CBBSI-Checklist based box system intervention, BpCr-Birth Preparedness and complication readiness, DsK-Danger Signs Knowledge)

knowledge of danger signs of labor and delivery and family support variables were treated as a latent variable in this analysis. For these latent variables, factor analysis was computed and factor reduction was done based on the factor loadings, taking 0.4 as a cutoff point. Then the relationship between each of the constructs with the latent variable was measured using generalized response confirmatory factor analysis. Similarly, factor analysis was also computed for social support, which was measured using a 14 item questions, a similar cut off point was applied.

Since this study contained a dependent variable 'institutional delivery' and various independent variables with generalized responses, Generalized Structural Equation Modeling (GSEM) was employed to examine the relationship between various exogenous and endogenous or mediating variables indicated in the hypothesized model (Fig. 1). This model was selected over multiple logistic regression models, because, logistic regression yields the direct effect of predictors on the outcome, but there might be variables that have a mediation effect (mediators) on the outcome.

Accordingly, institutional delivery, CBBSI, antenatal care four visit, month of ANC initiation, place of residence and influence of significant others had binary response categories and analyzed with Bernoulli family and logit link function. Social support was measured using 14 item questions. Initially social support was considered as a latent variable in the GSEM model. However, this increased model complexity and took longer time to converge. So, to get a model that best fits with the data, we compared two models: a model with social support as a latent variable and social support as a continuous variable. Social support was considered as a continuous variable through computing a raw sum scores, which produce a valid value ranging from 2 to 14 . As the number of parameters to be estimated were reduced, considering social support as a continuous predictor produces a relatively less complex model. Then, social support was analyzed with Gaussian family and identity link function. Birth preparedness and complication readiness practice, knowledge of labor and delivery danger signs and family support were latent variables which constitute items with binary response categories, their measurement model was analyzed with Bernoulli family and logit link function. Age was treated as a continuous variable in the GSEM. Age and social support were categorized for the purpose of descriptive report.

The analysis was started with fitting the hypothesized model, and modifications were performed through adding theoretically supported path links. Finally a model with the minimum Akaike information criteria was retained. After the parameters were estimated, an indirect and total effects were computed through a nonlinear combination of parameters [30]. The mediation effect was reported as complete (insignificant direct pathway), partial (significant indirect path) and no mediation (insignificant indirect pathways).

\section{Result}

\section{Socio-demographic characteristics}

Data from 1062 mothers who gave birth 1 year before the survey were included in the analysis (Fig. 3). The majority of participants were from rural residence 886 (83.4\%), attended primary education $623(58.7 \%)$ and were in marital union 1020 (96.1\%) (Table 1).

\section{Effectiveness of checklist based box system intervention on improving institutional delivery}

Of the participants, $432(85.2 \%)$ mothers from intervention and 287 (51.7\%) from control achieved antenatal 


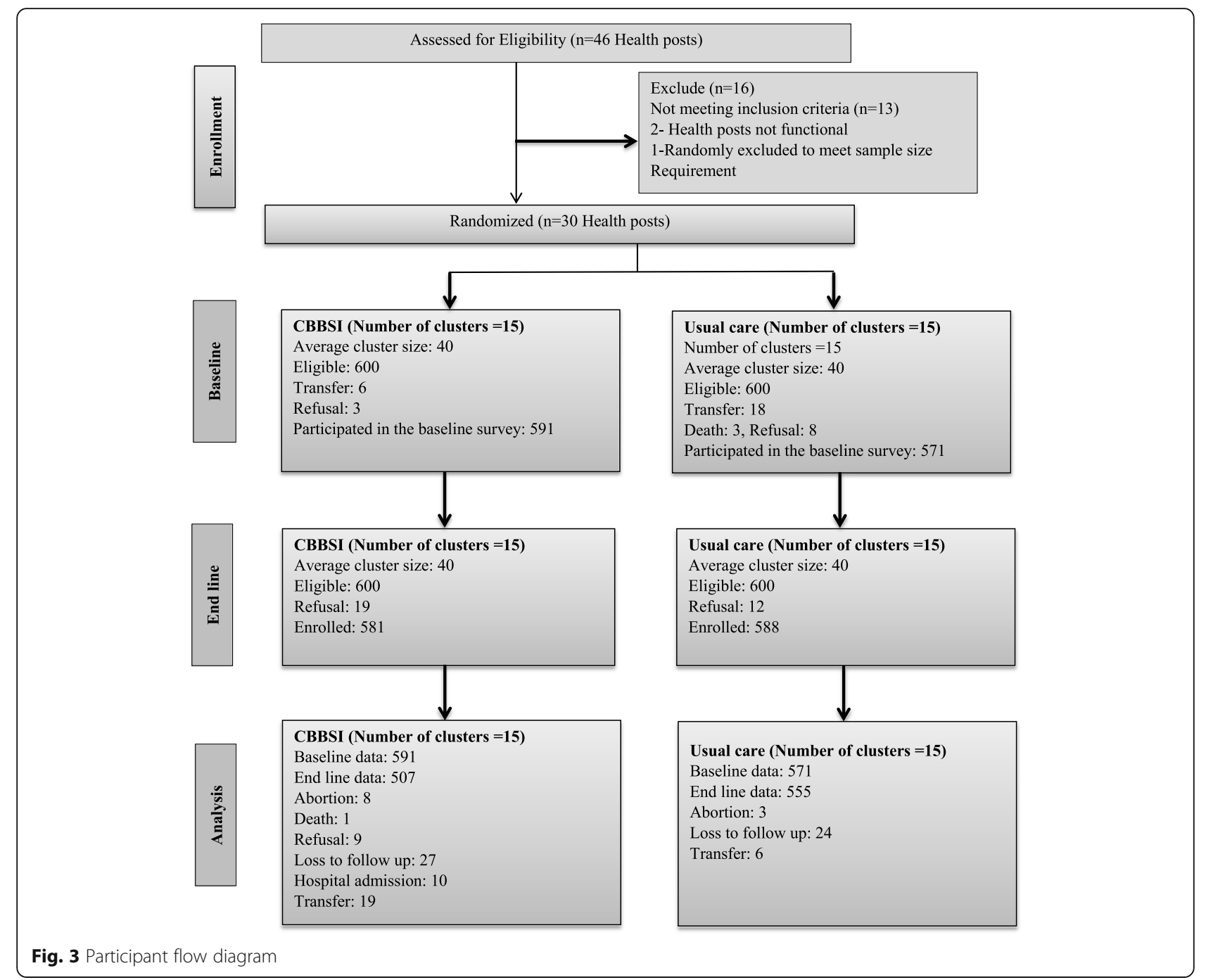

care four visits for their index pregnancy. Similarly, 403 (79.5\%) mothers from intervention and 323 (58.2\%) from control gave birth at health facilities (Table 2).

The difference-in-difference estimator showed that checklist-based box system intervention significantly improved institutional delivery in the study area (19, 95\%CI: 11.4-27.3\%). However, the lower confidence limit is slightly below the minimum difference that was planned to be detected between the intervention and control clusters, which was (12\%).

\section{Factors associated with institutional delivery}

The factor loadings for the whole measurement model turned significant at $p$ value $<0.001$ (Table 3 ), which showed the significance of items in measuring their respective latent constructs.

The estimated path coefficients from the generalized structural equation model were showed in Fig. 4. In addition, path coefficients along with their $p$-values and confidence intervals were indicated in Table 4. Though the magnitude varies across paths, the finding of this study showed that the intervention was linked both through direct and indirect paths to institutional delivery.

Of the significant direct paths estimated, checklist based box system intervention accounted higher than the other path coefficients that are linked to institutional delivery, $(\mathrm{AOR}=3.32,95 \% \mathrm{CI}: 2.36-4.66)$ followed by antenatal care four visits $(\mathrm{AOR}=1.39,95 \% \mathrm{CI}: 1.02-1.92)$ and social supports (AOR $=1.27,95 \% \mathrm{CI}$ : 1.15-1.40).

However, from the direct paths, age of the mother $(\mathrm{AOR}=0.03,95 \% \mathrm{CI}: 0.01-0.09)$ and influence of significant others (mother, grandmother, mother, neighbors, and husband) on the process of using maternal health services $(\mathrm{AOR}=1.28,95 \% \mathrm{CI}: 1.16-1.54)$ showed an inverse relationship with institutional delivery.

Indirect paths to institutional delivery from the intervention through BPCRp, knowledge of danger signs of 
Table 1 Description of Socio-demographic characteristics, January 2019-September 2020, Northwest Ethiopia

\begin{tabular}{|c|c|c|c|c|c|c|}
\hline \multirow[t]{2}{*}{ Variable } & \multicolumn{2}{|c|}{ Intervention $(n=507)$} & \multicolumn{2}{|c|}{ Control $(n=555)$} & \multirow[t]{2}{*}{$x^{2}$} & \multirow[t]{2}{*}{$p$-value } \\
\hline & Freq. & $\%$ & Freq. & $\%$ & & \\
\hline Age & & & & & 11.16 & 0.004 \\
\hline $15-19$ & 10 & 1.9 & 29 & 5.2 & & \\
\hline $20-29$ & 352 & 69.4 & 345 & 62.2 & & \\
\hline $30-49$ & 145 & 28.6 & 181 & 32.6 & & \\
\hline Place of Residence & & & & & 60.24 & $<0.0001$ \\
\hline Rural & 376 & 74.2 & 510 & 91.9 & & \\
\hline Urban & 131 & 25.8 & 45 & 8.1 & & \\
\hline Educational Status & & & & & 32.34 & $<0.0001$ \\
\hline No formal Education & 115 & 22.7 & 169 & 30.5 & & \\
\hline Primary (1-8th grade) & 293 & 57.8 & 330 & 59.5 & & \\
\hline Secondary (9-12th grade) & 62 & 12.2 & 49 & 8.8 & & \\
\hline Above 12th Grade & 377 & 74.4 & 7 & 1.3 & & \\
\hline Marital Status & & & & & 1.06 & 0.79 \\
\hline Single & 6 & 1.2 & 10 & 1.8 & & \\
\hline Married & 490 & 96.6 & 530 & 95.5 & & \\
\hline Separated & 10 & 1.9 & 14 & 2.5 & & \\
\hline Widowed & 1 & 0.2 & 1 & 0.2 & & \\
\hline Wealth Index & & & & & 18.30 & 0.001 \\
\hline Poorest & 199 & 39.3 & 207 & 37.3 & & \\
\hline Poor & 144 & 28.4 & 173 & 31.2 & & \\
\hline Medium & 73 & 14.4 & 116 & 20.9 & & \\
\hline Rich & 64 & 12.6 & 46 & 8.3 & & \\
\hline Richest & 27 & 5.3 & 13 & 2.3 & & \\
\hline Parity & & & & & 2.10 & 0.34 \\
\hline One Child & 221 & 45.6 & 218 & 39.3 & & \\
\hline 2-4 Children & 237 & 46.7 & 282 & 50.8 & & \\
\hline$\geq 5$ Children & 49 & 9.7 & 55 & 9.9 & & \\
\hline
\end{tabular}

labor and delivery, month of ANC initiation, and ANC four visits were created. Similarly, social support was linked by indirect path links through month of ANC initiation and ANC four visits to institutional delivery. Of this indirect path links, the path from the intervention to antenatal care four was the highest significant path $(\mathrm{AOR}=5.64,95 \% \mathrm{CI}: 4.18-7.61)$, followed by the path from the month of ANC initiation to ANC 4 (AOR = 1.97, 95\%CI: 1.43-2.69). The indirect path from social support to month of ANC initiation $(\mathrm{AOR}=1.05$, 95\%CI: 0.97-1.14) and also from social support to knowledge of danger signs of labor and delivery $(\mathrm{AOR}=1.12$, 95\%CI: 1.09-1.14) turned significant (Fig. 4, Table 4).

The total effect of checklist based box system intervention on institutional delivery was $5.92(\mathrm{AOR}=5.92$, 95\%CI: 3.34-10.38). The direct component of this effect was3.32 (AOR $=3.32,95 \% \mathrm{CI}: 2.36-4.66)$. In addition, ANC 4 visit was found to be a mediator, $33 \%$ of the effect of the intervention on institutional delivery was partially mediated by antenatal care four attendance (Table 5).

\section{Discussion}

The result of this study showed that checklist-based box system intervention was found effective in improving institutional delivery. In addition, the pathways through which the intervention was linked were tested. Accordingly, the pathway between the intervention and institutional delivery was mainly direct. However, antenatal care four visit partially mediated the effect. The remaining indirect pathways turned insignificant. This study has a similar finding with other studies which showed the contribution of four antenatal care to institutional delivery $[14,15,22-24,27]$. When the frequency of contact between mothers and health care providers is increasing, health messages delivered during 
Table 2 Description of maternal health service utilization, January 2019-September 2020, Northwest Ethiopia (Intervention ( $n$ ) = 507, Control $(n)=555)$

\begin{tabular}{|c|c|c|c|c|c|c|}
\hline \multirow[t]{2}{*}{ Variable } & \multicolumn{2}{|c|}{ Intervention } & \multicolumn{2}{|c|}{ Control } & \multirow[t]{2}{*}{$\mathrm{X}^{2}$} & \multirow[t]{2}{*}{$p$-value } \\
\hline & Freq. & $\%$ & Freq. & $\%$ & & \\
\hline Institutional Delivery & & & & & 55.5 & $<0.0001$ \\
\hline Yes & 403 & 79.5 & 323 & 58.2 & & \\
\hline No & 104 & 20.5 & 232 & 41.8 & & \\
\hline Antenatal Care four Visit & & & & & 135.9 & $<0.0001$ \\
\hline Yes & 432 & 85.2 & 287 & 51.7 & & \\
\hline No & 75 & 14.8 & 268 & 48.3 & & \\
\hline Month of the first ANC Initiation & & & & & 0.98 & 0.32 \\
\hline$\leq 16$ weeks of gestation & 193 & 35.1 & 162 & 32.1 & & \\
\hline$>16$ weeks of gestation & 359 & 65.0 & 343 & 67.9 & & \\
\hline \multicolumn{7}{|l|}{ Level of Birth Preparedness } \\
\hline Arranged emergency transport (yes) & 290 & 57.2 & 342 & 61.6 & 2.15 & 0.14 \\
\hline Personal saving and how to access them (yes) & 258 & 50.9 & 297 & 53.5 & 0.73 & 0.39 \\
\hline Arranged emergency funds (yes) & 272 & 53.6 & 279 & 50.3 & 1.21 & 0.27 \\
\hline Identify place of delivery/skilled attendant (yes) & 214 & 42.2 & 181 & 32.6 & 10.44 & 0.001 \\
\hline Knows who the blood donor is (yes) & 236 & 46.5 & 265 & 47.7 & 0.15 & 0.69 \\
\hline \multicolumn{7}{|l|}{ Knowledge of Danger signs of labor and Delivery } \\
\hline Severe headache (yes) & 473 & 93.2 & 522 & 94.1 & 0.26 & 0.61 \\
\hline Vaginal bleeding (yes) & 356 & 70.2 & 299 & 53.9 & 29.94 & $<0.0001$ \\
\hline Convulsion (yes) & 240 & 47.3 & 271 & 48.8 & 0.24 & 0.63 \\
\hline High Fever (yes) & 235 & 46.4 & 281 & 50.6 & 1.94 & 0.16 \\
\hline Loss of Consciousness (yes) & 244 & 48.1 & 245 & 44.1 & 1.69 & 0.19 \\
\hline Retained Placenta (yes) & 224 & 44.2 & 212 & 38.2 & 3.91 & 0.04 \\
\hline Prolonged labor (yes) & 254 & 50.1 & 299 & 53.9 & 1.51 & 0.23 \\
\hline \multicolumn{7}{|l|}{ Family Support } \\
\hline Provided advise/support to deliver at health facilities (yes) & 356 & 70.2 & 321 & 57.8 & 1.17 & 0.28 \\
\hline Help to get emergency transport during labor (yes) & 321 & 63.3 & 368 & 66.3 & 3.64 & 0.05 \\
\hline Accompany during labor (yes) & 292 & 57.6 & 328 & 59.1 & 1.47 & 0.22 \\
\hline Help to care my baby (yes) & 402 & 79.3 & 358 & 64.5 & 24.91 & $<0.0001$ \\
\hline Help to attend post-natal visits (yes) & 261 & 51.5 & 294 & 52.9 & 1.22 & 0.27 \\
\hline Level of Social Support & & & & & 3.10 & 0.07 \\
\hline Good & 344 & 67.9 & 404 & 72.8 & & \\
\hline Poor & 163 & 32.1 & 151 & 27.2 & & \\
\hline Influence of Significant others & & & & & 9.22 & 0.002 \\
\hline Yes & 60 & 11.8 & 36 & 6.5 & & \\
\hline No & 447 & 88.2 & 519 & 93.5 & & \\
\hline
\end{tabular}

counseling and health education sessions increased and mothers will get enough time to internalize the messages delivered by health care providers. Though most of the association between the intervention and institutional delivery was direct, antenatal care four attendance was found a mediator trough the path to institutional delivery. This could indicate the importance of the continuum of care approach while designing maternal health interventions.

In Ethiopia, m-health interventions and educational messages were used in one of the interventional study aimed at improving maternal health utilization through a continuum of care approach [19]. The other two interventions aimed to increase institutional delivery utilization, the first by improving maternity waiting 
Table 3 Confirmatory Factor Analysis: unstandardized factor loadings, January 2019-September 2020, Northwest Ethiopia (N=1062)

\begin{tabular}{|c|c|c|}
\hline Constructs and indicators & Unstandardized factor loadings & $p$-value \\
\hline Level of Birth Preparedness & & Cronbatch alpha $=0.88$ \\
\hline $\mathrm{BpCr} 1$ - Arranged emergency transport & 1 & \\
\hline BpCr2 - Personal saving and how to access them & 1.15 & $<0.0001$ \\
\hline $\mathrm{BpCr} 3$ - Arranged emergency funds & 2.28 & $<0.0001$ \\
\hline BpCr4 - Identify place of delivery/skilled attendant & 0.63 & $<0.0001$ \\
\hline $\mathrm{BpCr} 5$ - Knows who the blood donor is & 1.72 & $<0.0001$ \\
\hline Knowledge on labor and delivery danger signs & & Cronbatch alpha $=0.89$ \\
\hline DsK2 - Vaginal bleeding & 1 & \\
\hline DsK3 - Convulsion & 1.73 & $<0.0001$ \\
\hline DsK4 - High Fever & 1.53 & $<0.0001$ \\
\hline DsK5 - Loss of Consciousness & 1.17 & $<0.0001$ \\
\hline DsK6 - Retained Placenta & 0.68 & $<0.0001$ \\
\hline DsK7 - Prolonged labor & 1.31 & $<0.0001$ \\
\hline Family Support & & Cronbatch alpha $=0.85$ \\
\hline Fs1 - Provided advise/support to deliver at health facilities & 1 & \\
\hline Fs2 - Help to get emergency transport during labor & 1.04 & $<0.0001$ \\
\hline Fs3 - Accompany during labor & 2.31 & $<0.0001$ \\
\hline Fs5 - Help to attend post-natal visits & 0.19 & $<0.0001$ \\
\hline
\end{tabular}

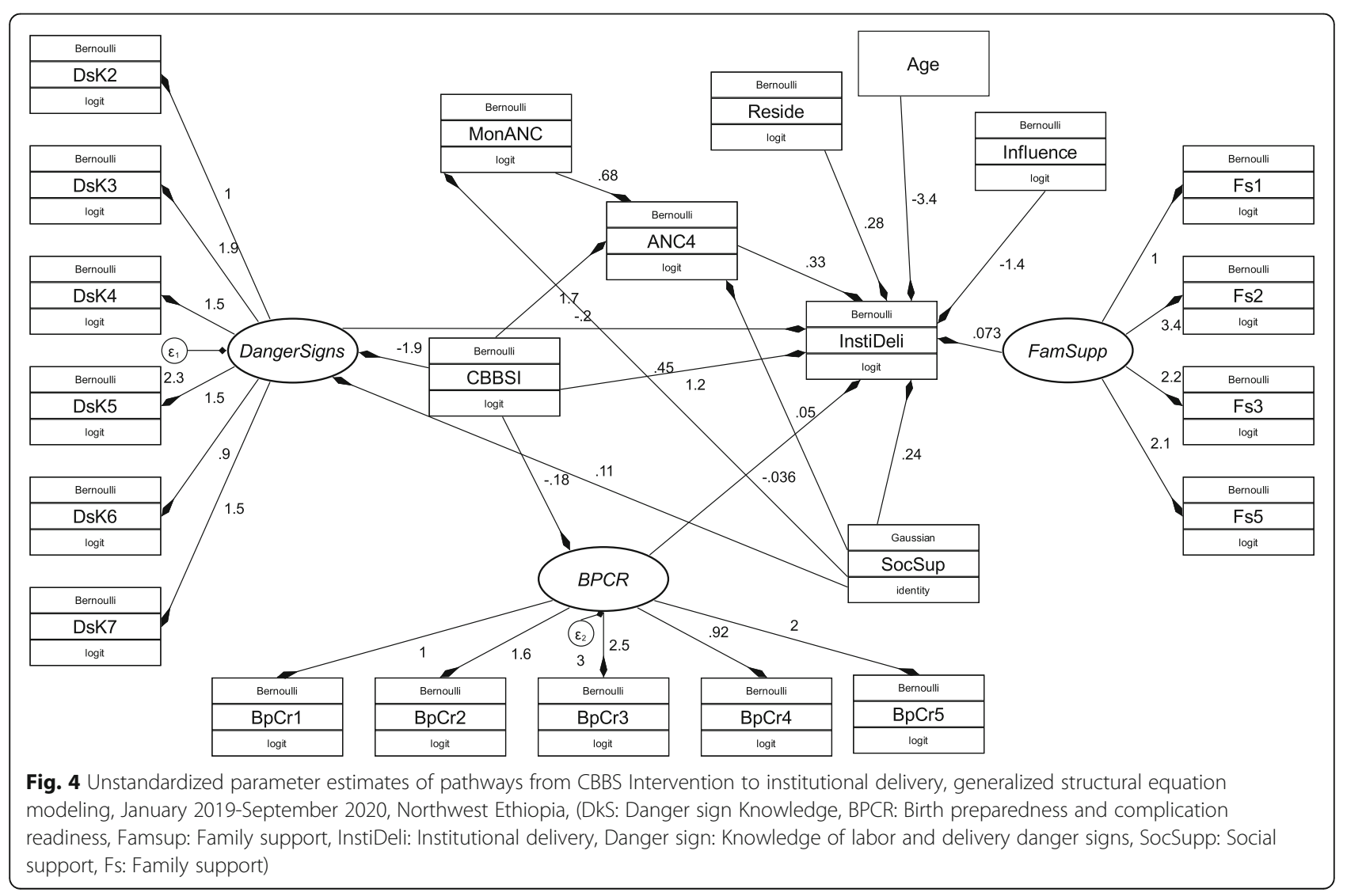


Table 4 Adjusted odds ratios from the generalized structural equation modeling, January 2019-September 2020, Northwest Ethiopia

\begin{tabular}{|c|c|c|}
\hline Structural model & AOR & $(95 \% \mathrm{Cl})$ \\
\hline \multicolumn{3}{|l|}{ Outcome: Institutional delivery } \\
\hline \multicolumn{3}{|c|}{ Checklist based box system intervention } \\
\hline Control (Ref) & - & - \\
\hline Intervention & $3.32^{*}$ & $2.36-4.66$ \\
\hline \multicolumn{3}{|l|}{ ANC four visit } \\
\hline No (Ref) & - & - \\
\hline Yes & $1.39^{*}$ & $1.02-1.92$ \\
\hline Danger Signs & 0.83 & $0.63-1.08$ \\
\hline BPCRp & 0.96 & $0.78-1.19$ \\
\hline Social Support & $1.27^{*}$ & $1.15-1.40$ \\
\hline Family Support & 1.07 & $0.93-1.25$ \\
\hline \multicolumn{3}{|l|}{ Influence } \\
\hline No (Ref) & - & - \\
\hline Yes & $1.28^{*}$ & $1.16-1.54$ \\
\hline Age & $0.03^{*}$ & $0.01-0.09$ \\
\hline \multicolumn{3}{|l|}{ Place of Residence } \\
\hline Rural (Ref) & - & - \\
\hline Urban & 1.32 & $0.80-2.16$ \\
\hline \multicolumn{3}{|c|}{ Indirect path: Antenatal care 4 attendance } \\
\hline Control (Ref) & - & - \\
\hline Intervention & $5.64^{*}$ & $4.18-7.61$ \\
\hline \multicolumn{3}{|l|}{ Month of ANC Initiation } \\
\hline$>16$ weeks of gestation (Ref) & - & - \\
\hline$\leq 16$ weeks of gestation & $1.97^{*}$ & $1.43-2.69$ \\
\hline Social support & 1.05 & $0.97-1.14$ \\
\hline \multicolumn{3}{|l|}{ Indirect path: BPCRp } \\
\hline Control (Ref) & - & - \\
\hline Intervention & 0.84 & $0.70-1.04$ \\
\hline \multicolumn{3}{|l|}{ Indirect path: Danger signs } \\
\hline Control (Ref) & - & - \\
\hline Intervention & 0.15 & $0.11-5.05$ \\
\hline \multicolumn{3}{|c|}{ Indirect Path: Month of ANC Initiation } \\
\hline Social Support & $1.57^{*}$ & $1.45-1.69$ \\
\hline \multicolumn{3}{|l|}{ Indirect path: Danger signs } \\
\hline Social Support & $1.12^{*}$ & $1.09-1.14$ \\
\hline
\end{tabular}

$I D$ institutional delivery, $C B B S I$ Checklist based box system intervention, $B P C R$ Birth preparedness and complication readiness ${ }^{*} p<0.05$

home utilization [18] and the second by deploying trained community-based nurses to rural communities [20]. The former was implemented m-health interventions in order to increase maternal health service utilization (ANC, institutional delivery and PNC). However, most mothers, particularly in rural Ethiopia, did not own mobile phones. Using CBBSI could be beneficial in this regard because it does not necessitate infrastructure, telecom networking, or the ownership of mobile phones. The latter two interventions, which focus solely on improving institutional delivery, didn't take the continuum of care approach into consideration.

In this study, there was no observed effect of the intervention through birth preparedness and complication readiness practice and knowledge of labor and delivery danger signs. However, there are studies that showed, the positive effect of both birth preparedness practice [31] and knowledge of labor and delivery danger signs on utilization of institutional delivery $[8,29]$. These discrepancies might be because of the nature and approaches of the study and difference while applying the measurement tools. This intervention has both demand creation and service utilization monitoring components. The above finding could indicate that, service utilization component of the intervention, which was implemented through drop-out tracing mechanisms, contributed more for institutional delivery through increasing ANC 4 attendances than the demand creation component. In addition, this study measured BPCR practice of mothers, not the knowledge component. The intervention might have an effect on improving the knowledge of mothers on things that should be fulfilled while preparing for labor and delivery, but the practice is always considered as the effort of mothers.

Social support was found to have a positive and significant relation with knowledge of danger signs of labor and delivery. Similarly, the study showed a positive and significant relationship between social support and institutional delivery. This is in agreement with a study conducted in Kersa, Ethiopia [24], which recommend the importance of social support structures for improving institutional delivery. In addition, an indirect effect of social support was observed through improving early initiation of antenatal care (before 16 weeks of gestation). This in turn improves frequency of ANC visits. Hence, the use of community level social structures would have a paramount importance in disseminating health related messages. The intervention could also be benefited, if the community level demand creation activities could be reached through an identified and functional social structures.

Other determinants of maternal health service utilization, such as age and influence of significant others have also showed a direct but inverse relation with institutional delivery. In the study area, going to health facilities for childbirth service was considered as a lack of internal strength of a woman, especially by older generation of individuals (mother, mother in-law, grandmother and husband) who probably influence the decision of a mother. So, extending demand creation 
Table 5 Direct, indirect and total effect of CBBSI on institutional delivery, January 2019-September 2020, Northwest Ethiopia

\begin{tabular}{llll}
\hline Outcome: institutional delivery & AOR (95\% CI) & & $\boldsymbol{p}$-value \\
\hline CBBS Intervention & & $5.92(3.34-10.38)$ & $<0.001$ \\
Total effect & & $3.32(2.36-4.66)$ & $<0.001$ \\
Direct effect & Antenatal Care Four attendance & $1.79(1.02-3.13)$ & 0.04 \\
Indirect effects via & Knowledge of Labor and delivery danger signs & $1.45(0.87-2.43)$ & 0.89 \\
& Birth preparedness and complication readiness practice & $1.01(0.97-1.04)$ & 0.75 \\
\hline
\end{tabular}

activities to reach to significant others, who might have a decisive role on the life of a mothers could contribute indirectly.

\section{Strength and limitation}

The study used a double-blind approach, in which mothers and outcome assessors were unaware of the intervention, which groups belonged to the intervention and control groups, and the hypothesis that would be tested. In these process, the study limits the occurrence of ascertainment bias or detection bias. Furthermore, during analysis of the effect size, the study's use of simple randomization procedures was taken into account by employing difference-in-difference estimation. However, because the utilization of maternal health services was self-reported, there is a possibility of self-reporting or recall bias as a limitation.

\section{Sustainability}

The checklist-based box system intervention was built on the government's existing health system structure, in which health posts are linked to nearby health centers. In addition, the intervention was carried out using the country's government-owned flagship program, the health extension program (which is being implemented throughout the country). In this regard, the authors believe that the intervention has a high likelihood of being sustainable. However, more research on the feasibility, sustainability, and adaptability of the intervention is needed.

\section{Conclusion}

Checklist based box system intervention was found effective in improving institutional delivery. The pathway through which the intervention was linked with institutional delivery was mainly direct, however, it was partially mediated by antenatal care four visits. In addition, social support and month of initiation of ANC showed a positive relation with institutional delivery. In the contrary, age and influence of significant other inversely related with institutional delivery. The implementation of checklist based box system intervention need to consider the synergetic and complementary effects of maternal health services on each other. Also, this intervention could be re-designed to incorporate functional community level structures for demand creation activities.

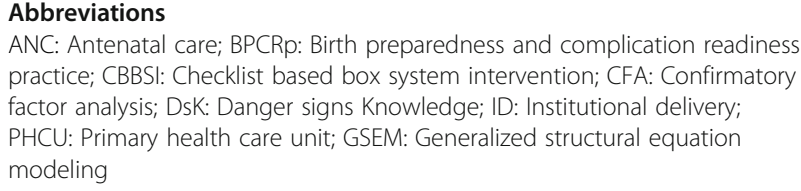
practice; CBBSI: Checklist based box system intervention; CFA: Confirmatory factor analysis; DsK: Danger signs Knowledge; ID: Institutional delivery; PHCU: Primary health care unit; GSEM: Generalized structural equation modeling

\section{Supplementary Information}

The online version contains supplementary material available at https://doi. org/10.1186/s13690-021-00774-2

\section{Additional file 1.}

Additional file 2

\section{Acknowledgements}

We would like to thank the Grand challenges Ethiopia of the Armauer Hansen Research Institute for providing financial support for the study. We are also grateful for the contribution of Amhara public Health Institute, East Gojjam zone health department, and respective implementation districts, health care providers, health extension workers, study participants supervisors and data collectors for their cooperation and facilitation while conducting this study.

\section{Authors' contributions}

NBA, initiated the trial idea, wrote the initial draft of the proposal, developed the protocol and supervised the implementation of the intervention and wrote the first draft manuscript. NBA and GTD participated in study design, designing intervention plan, data acquisition, and data analysis and reviewing the manuscript. Both authors critically reviewed and approved the final manuscript.

\section{Funding}

This study is part of a PhD research work of the corresponding author, under population and family health department of Jimma University. Her project was funded by Armauer Hansen Research Institute (AHRI). AHRI has no role in the design of the study and data collection, analysis, and interpretation of data and in writing manuscripts.

\section{Availability of data and materials}

The datasets used and/or analyzed during the current study are available from the corresponding author on reasonable request.

\section{Declarations}

Ethics approval and consent to participate

The proposal was reviewed and approved by Jimma University, institute of health- institutional review board (IHRPGD/841/17) and Amhara region public health institute. Then the purpose of the study was briefly explained for the respondents and oral informed consent was maintained. Each and every interviewee has their own identification code. During data collection, study participants were well informed that the information collected was protected and kept anonymous and confidential. 


\section{Consent for publication}

Not Applicable.

\section{Competing interests}

The authors declare that they have no competing interests.

Received: 12 July 2021 Accepted: 17 December 2021

Published online: 04 January 2022

\section{References}

1. WHO. Recommendations on maternal health. The future of eco-labelling: making environmental product information systems effective. 2017.

2. WHO. Making pregnancy safer: a strategy for action. Safe Motherhood Newsletter. 2009.

3. Kwast BE, Liff JM. Factors associated with maternal mortality in Addis Ababa, Ethiopia. Int J Epidemiol. 1988;17(1):115-21.

4. Ekele BA, Tunau KA. Place of delivery among women who had antenatal care in a teaching hospital. Acta Obstet Gynecol Scand. 2007;86(5):627-30.

5. Graham H, Bell J, Bullough C. Can skilled attendance at delivery reduce maternal mortality in developing countries? Safe motherhood strategies a review of the evidence; 2001

6. Darmstadt GL, Bhutta ZA, Cousens S, Adam T, Walker N, De Bernis L. Evidence-based, cost-effective interventions: how many newborn babies can we save? Lancet. 2005:365(9463):977-88.

7. UNICEF. The state of the world's children: maternal and newborn health New York: WHO; p. 1-168. 2009.

8. Ayele GS, Melku AT, Belda SS. Utilization of skilled birth attendant at birth and associated factors among women who gave birth in the last 24 months preceding the survey in Gura Dhamole Woreda, Bale zone, Southeast Ethiopia. BMC Public Health. 2019;19(1):1-14.

9. CSA. Ethiopia mini demographic and health survey: key indicators. 2019.

10. Haruna U, Dandeebo G, Galaa SZ. Improving access and utilization of maternal healthcare services through focused antenatal care in Rural Ghana: a qualitative study. Adv Public Health. 2019;2019:1-11.

11. Ministry of Health and Social Welfare, Tanzania. Focused antenatal care malaria and syphilis in pregnancy. Learner's Guide for ANC Service Providers and Supervisors. 2009

12. Hailu M, Gebremariam A, Alemseged F, Deribe K. Birth preparedness and complication readiness among pregnant women in Southern Ethiopia. PLoS One. 2011;6(6):e21432.

13. Anshebo D, Geda B, Mecha A, Liru A, Id RA. Utilization of institutional delivery and associated factors among mothers in Hosanna Town, Hadiya Zone, Southern study; 2020. p. 1-12. Available from: https://doi.org/10.1371/ journal.pone.0243350

14. Mitikie KA, Wassie GT, Beyene MB. Institutional delivery services utilization and associated factors among mothers who gave birth in the last year in Mandura district, Northwest Ethiopia. PLoS ONE.2020;15(12):e0243466. https://doi.org/10.1371/journal.pone.0243466.

15. Bayu H, Fisseha G, Mulat A, Yitayih G, Wolday M. Missed opportunities for institutional delivery and associated factors among urban resident pregnant women in South Tigray Zone, Ethiopia: a community-based follow-up study. Global Health Action. 2015;8:1-8.

16. Nigatu AM, Gelaye KA. Factors associated with the preference of institutional delivery after antenatal care attendance in Northwest Ethiopia. BMC Pregnancy and Childbirth. 2019;19:810.

17. Federal Ministry of Health Ethiopia(FMoH). Health sector transformation plan -1; 2017. p. 142

18. Kurji J, Gebretsadik LA, Wordofa MA, Morankar S, Bedru KH, Bulcha G, et al. Effectiveness of upgraded maternity waiting homes and local leader training on improving institutional births : a cluster- randomized controlled trial in Jimma; 2020. p. 1-15.

19. Shiferaw S, Spigt M, Tekie M, Abdullah M, Fantahun M, Dinant GJ. The effects of a locally developed mHealth intervention on delivery and postnatal care utilization; a prospective controlled evaluation among health centres in Ethiopia. PLoS One. 2016;11(7):1-14

20. Zerfu TA, Taddese H, Nigatu T, Tenkolu G, Khan DN, Biadgilign S, et al. Is deployment of trained nurses to rural villages a remedy for the low skilled birth attendance in Ethiopia? A cluster randomized-controlled community trial. PLoS One. 2018;13(10):1-12.

21. Andargie NB, Jebena MG, Debelew GT. Effectiveness of checklist-based box system interventions (CBBSI) versus routine care on improving utilization of maternal health services in Northwest Ethiopia: study protocol for a cluster randomized controlled trial. Trials. 2020:21(1):1-9.

22. Asefa A, Gebremedhin S, Messele T, Letamo Y, Shibru E, Alano A, et al. Mismatch between antenatal care attendance and institutional delivery in south Ethiopia: a multilevel analysis; 2019. p. 1-10.

23. Fekadu A, Yitayal M, Alemayehu GA, Abebe SM, Ayele TA, Tariku A, et al. Frequent antenatal care visits increase institutional delivery at Dabat health and demographic surveillance system site, Northwest Ethiopia; 2019. p. 2019

24. Tesfaye G, Chojenta C, Smith R, Loxton D. Predisposing, enabling and need factors associated with skilled delivery care utilization among reproductiveaged women in Kersa district, eastern Ethiopia. Reprod Health. 2019;16(1):111.

25. Ethiopian Demography and Zones, Amhara Regional State. http://www. ethiodemographyandhealth.org/Amhara.html.

26. Kumar K. A beginner's guide to structural equation modeling, 3rd edn. J R Stat Soc Ser A Stat Soc. 2012;175:828-9.

27. Fekadu GA, Kassa GM, Berhe AK, Muche AA, Katiso NA. The effect of antenatal care on use of institutional delivery service and postnatal care in Ethiopia: a systematic review and meta-analysis; 2018. p. 1-11.

28. Asseffa NA, Bukola F, Ayodele A. Determinants of use of health facility for childbirth in rural Hadiya zone, Southern. BMC Pregnancy Childbirth. 2016; 16(335):1-9.

29. Yoseph M, Abebe SM, Mekonnen FA, Sisay M, Gonete KA. Institutional delivery services utilization and its determinant factors among women who gave birth in the past 24 months in Southwest Ethiopia. BMC Health Serv Res. 2020;20(1):1-10

30. Id RS. Does delivery in private hospitals contribute largely to caesarean section births? A path analysis using generalised structural equation modelling; 2020. p. 1-24. Available from: https://doi.org/10.1371/journal. pone.0239649

31. Tura G, Afework MF, Yalew AW. The effect of birth preparedness and complication readiness on skilled care use: a prospective follow-up study in Southwest Ethiopia. Reproductive Health. 2014;11(1):1-10.

\section{Publisher's Note}

Springer Nature remains neutral with regard to jurisdictional claims in published maps and institutional affiliations.

Ready to submit your research? Choose BMC and benefit from:

- fast, convenient online submission

- thorough peer review by experienced researchers in your field

- rapid publication on acceptance

- support for research data, including large and complex data types

- gold Open Access which fosters wider collaboration and increased citations

- maximum visibility for your research: over $100 \mathrm{M}$ website views per year

At $\mathrm{BMC}$, research is always in progress.

Learn more biomedcentral.com/submissions 